Pacific Journal of Mathematics

NORM ATTAINING OPERATORS ON SOME CLASSICAL 


\title{
NORM ATTAINING OPERATORS ON SOME CLASSICAL BANACH SPACES
}

\author{
WALTER SCHACHERMAYER
}

\begin{abstract}
We construct an operator from $L^{1}[0,1]$ to $C[0,1]$ which may not be approximated by norm attaining operators with respect to the operator norm. This solves a question raised by J. Johnson and J. Wolfe and furnishes the first example of a pair of classical Banach spaces such that the norm attaining operators are not dense. $C[0,1]$ is the first example of a classical Banach space which does not have property $B$.

On the other hand, we show that a weakly compact operator from $C(K)$ into a Banach space $X$ may be approximated in norm by norm attaining operators. This shows in particular that the norm attaining operators are dense in $B\left(C(K), L^{1}[0,1]\right)$ and $B\left(C(K), l^{2}\right)$, thus solving two questions raised by Johnson and Wolfe.
\end{abstract}

Let $X, Y$ be Banach spaces, $B(X, Y)$ the Banach space of bounded linear operators $A$ from $X$ to $Y$ and $N A(X, Y)$ the subset of norm attaining operators $A$, i.e. there is $x \in X,\|x\|=1$ with $\|A x\|=\|A\|$. The question of density of $N A(X, Y)$ in $B(X, Y)$ was studied in [1], [3], [4], [5], [7] and [8]. As regards classical Banach spaces it was shown in [3] that $N A\left(L^{1}(\mu), L^{1}(\nu)\right)$ is dense in $B\left(L^{1}(\mu), L^{1}(\nu)\right)$ and it was shown in [4] that $N A(C(K), C(L))$ is dense in $B(C(K), C(K))$, but the cases treated in the present paper were left open.

After a preparatory part 1 we shall construct in part 2 an example from which the subsequent result follows

TheOREM A. $N A\left(L^{1}[0,1], C[0,1]\right)$ is not dense in $B\left(L^{1}[0,1], C[0,1]\right)$.

Finally, in part 3, which may be read independently of part 1 and 2 we prove

THEOREM B. Let $K$ be a compact Hausdorff space, $X$ a Banach space and $A: C(K) \rightarrow X$ a weakly compact operator. Then $A$ may be approximated in the operator norm by elements of $N A(C(K), X)$.

Hence, if $X$ does not contain $c_{0}$ isomorphically (in particular if $X=L^{1}(\mu)$ or $\left.X=l^{2}\right), N A(C(K), X)$ is dense in $B(C(K), X)$.

Our notation will be standard. $L^{1}$ will denote the usual Lebesgue space over $[0,1]$ equipped with Lebesgue measure, $T$ the compact space $[0,1]$ and $C$ the Banach space of continuous functions on $T$. Our Banach spaces will be real. 
1. Denote $\sigma^{*} C\left(T, L^{\infty}\right)$ the Banach space of functions $F: T \rightarrow L^{\infty}$ which are continuous with respect to the $\sigma^{*}$-topology of $L^{\infty}$, equipped with the norm

$$
\|F\|=\sup \left\{\left\|F_{t}\right\|_{L^{\infty}}: t \in T\right\} .
$$

We shall need the following trivial but useful representation:

1.1. Proposition. The Banach spaces $B\left(L^{1}, C\right)$ and $\sigma^{*} C\left(T, L^{\infty}\right)$ are naturally isometrically isomorphic. The correspondence between $A \in$ $B\left(L^{1}, C\right)$ and $F \in \sigma^{*} C\left(T, L^{\infty}\right)$ is given by $F_{t}=A^{*}\left(\delta_{t}\right)$, where $A^{*}$ denotes the adjoint of $A$ and $\delta_{t}$ the Dirac measure at $T$.

$A$ is norm attaining iff there is $t \in T$ such that the function $\left|F_{t}\right|$ equals $\|F\|$ on a set of positive measure.

1.2. Hence the problem of whether we may approximate $A \in B\left(L^{1}, C\right)$, $\|A\|=1$, by $\tilde{A} \in N A\left(L^{1}, C\right)$ (with $\|\tilde{A}\|=1$ say) up to $\varepsilon$ is equivalent to the following question: Given $F \in \sigma^{*} C\left(T, L^{\infty}\right),\|F\|=1$, does there exist $\tilde{F} \in \sigma^{*} C\left(T, L^{\infty}\right),\|\tilde{F}\|=1$ and $\|F-\tilde{F}\|<\varepsilon$ such that $\tilde{F}$ hits at some point $t$ a norm attaining element of the unit sphere of $L^{\infty}$ (i.e. $\left|F_{t}\right|$ equals 1 on a set of positive measure)?

There is a natural guess for the construction of such a $\tilde{F}$ for a given $F$ with $\|F\|=1$ : By changing sign if necessary we may suppose that there is $t_{0} \in T$ such that $F_{t_{0}}$ is greater than $1-\varepsilon$ on a set of positive measure. Then

$$
\tilde{F}_{t}=\left(F_{t}+\varepsilon\right) \wedge 1
$$

defines a function $\tilde{F}$ into the unit ball of $L^{\infty}$ with $\left\|F_{t}-\tilde{F}_{t}\right\| \leq \varepsilon$ for $t \in T$ and such that $\tilde{F}_{t_{0}}$ equals 1 on a set of positive measure. The crux is, however, that it may happen that $\tilde{F}$ is not $\sigma^{*}$-continuous. Suppose for example that there is a sequence $\left(s_{n}\right)_{n=1}^{\infty}$ in $T$ tending to $s_{0}$ such that $F_{s_{n}}$ equals the $n$th Rademacher function $R_{n}$ (and therefore by $\sigma^{*}$-continuity $F_{s_{0}}=0$ ). Then for the $\tilde{F}$ constructed above $\left(\tilde{F}_{s_{n}}\right)$ is a sequence of independent random variables taking the values 1 and $-1+\varepsilon$ with probability $1 / 2$ and so converges $\sigma^{*}$ to the constant function $\varepsilon / 2$. However, $\tilde{F}_{s_{0}}$ equals the constant function $\varepsilon$, i.e. $\tilde{F}$ is not $\sigma^{*}$-continuous. It will be this phenomenon on which our example is essentially based.

But it will be instructive to investigate this idea a little further in order to get some information and motivation, how to construct our example. On the unit ball $O L^{\infty}$ of $L^{\infty}$ we also have the topology of the $L^{1}$-norm, which is finer than the $\sigma^{*}$-topology and for which $O L^{\infty}$ is a complete metric space. Suppose that $F \in \sigma^{*} C\left(T, L^{\infty}\right),\|F\|=1$ is continuous with respect to the $L^{1}$-topology on $O L^{\infty}$. Then it is easy to check that $\tilde{F}=$ $(F+\varepsilon) \wedge 1$ is also continuous with respect to the $L^{1}$-norm, so a fortiori $F \in \sigma^{*} C\left(T, L^{\infty}\right)$. 
Hence $A \in B\left(L^{1}, C\right)$ is approximable by norm attaining operators if the corresponding $F$ is continuous with respect to the $L^{1}$-norm on $L^{\infty}$.

Let us still note that this latter class of operators is strictly larger than the class of Riesz-representable operators from $L^{1}$ to $C$ (for definition see [2]). Indeed, it is easy to check that $A$ is Riesz-representable iff for $t_{n} \rightarrow t_{0}$ we have that $F_{t_{n}}$ converges almost everywhere to $F_{t_{0}}$. Note that for bounded sequences in $L^{\infty}$ the almost sure convergence is strictly stronger than the convergence with respect to the $L^{1}$-norm. Hence the class of operators $A$ such that the corresponding $F$ is $\|\cdot\|_{1}$-continuous includes the class of Riesz-respresentable operators, and-after the above remarks-it is not difficult to give examples showing that the inclusion is strict. Hence the above observation gives, for the special situation of operators from $L^{1}$ to $C$, a slightly more general result than ([8], th. 2$)$.

To finish the introductory part of the paper, we shall show that for arbitrary $F \in \sigma^{*} C\left(T, L^{\infty}\right)$ there are "many" points of $T$ at which $F$ is $\|\cdot\|_{1}$-continuous. This shows the limitations for the subsequent construction of a counterexample: The above described phenomenon can only occur at "few" points.

1.3. Proposition. Let $F \in \sigma^{*} C\left(T, L^{\infty}\right)$. There is a dense $G_{\delta}$-set $M \subseteq T$ such that, at every point of $M, F$ is continuous with respect ot the $L^{1}$-norm.

Proof. Let

$$
K=\left\{f \in L^{1}:\|f\|_{\infty} \leq 1\right\}
$$

which is compact if equipped with the weak topology of $L^{1}$. Define

$$
\begin{aligned}
G: T \times K & \rightarrow \mathbf{R} \\
(t, f) & \rightarrow\left\langle F_{t}, f\right\rangle
\end{aligned}
$$

where $\langle\cdot, \cdot\rangle$ denotes the scalar product between $L^{\infty}$ and $L^{1}$. Clearly $G$ is separably continuous. It follows from ([6], th. 1.2) that there is a dense $G_{\delta}$-set $M$ in $T$ such that, for $t \in M$ and $f \in K, G$ is jointly continuous at $(t, f)$.

In view of the compactness of $K$ this implies that if $\left(t_{n}\right)_{n=1}^{\infty} \subseteq T$ tends to some $t \in M$, then $F_{t_{n}}$ tends to $F_{t}$ uniformly on $K$, i.e. $F_{t_{n}}$ tends to $F_{t}$ in the $L^{1}$-norm.

2. We now proceed to the proof of Theorem A. It will be convenient to introduce an ad-hoc-concept. 
2.1. Definition. Let $F \in \sigma^{*} C\left(T, L^{\infty}\right),\|F\| \leq 1$ and $S$ be a subset of $T$. For $r \in T, F$ is called "hopeless at $r$ " (resp. " $S$-hopeless at $r$ ") if

(a) $F_{r}=\sum_{i=1}^{n}\left(1-\lambda_{i}\right) \chi_{A_{i}}$ for some partition $\left(A_{i}\right)_{i=1}^{n}$ of $\Omega$ and scalars $0<\lambda_{i} \leq 1$, and

(b) for every $A$ contained in some $A_{i}$ and $\varepsilon>0$ there is $s \in T$ (resp. $s \in S)$ with $|r-s|<\varepsilon$ and $\mu\left\{\omega \in A:\left|F_{s}(\omega)\right| \leq \varepsilon\right\} \geq \mu(A) \cdot \lambda_{i} / 2$.

2.2. Proposition. Let $F \in \sigma^{*} C\left(T, L^{\infty}\right),\|F\| \leq 1$ be hopeless at some $r \in T$ and $\tilde{F} \in \sigma^{*} C\left(T, L^{\infty}\right)$ with $\|\tilde{F}\| \leq 1$ and $\|F-\tilde{F}\| \leq 1 / 2$. Then for $i=1, \ldots, n$,

$$
\operatorname{ess} \sup \left\{\tilde{F}_{r}(\omega): \omega \in A_{i}\right\} \leq 1-\lambda_{i} / 4 \text {. }
$$

In particular if $F$ satisfies in addition for every $n \in \mathbf{N}$

$$
\mu\left\{F_{r}>1-2^{-n}\right\} \leq \alpha_{n}
$$

then $\tilde{F}_{r}$ satisfies for every $n \in \mathbf{N}$

$$
\mu\left\{\tilde{F}_{r}>1-2^{-n-2}\right\} \leq \alpha_{n} .
$$

REMARK. The intuitive meaning of 2.2 and the reason for the concept of "hopelessness": If $F$ is hopeless at $r$ and the distribution of $F_{r}$ obeys the inequalities (2) and if $\tilde{F}$ is a perturbation of $F$, then the distribution of $\tilde{F}_{r}$ obeys (3) and $\tilde{F}_{r}$ therefore is a "hopeless candidate" for being a norm attaining element of the unit sphere of $L^{\infty}$.

Proof. For $A \subseteq A_{i}$ it follows immediately from Definition 2.1, the $\sigma^{*}$-continuity of $\tilde{F}$ and the assumption $\|\tilde{F}\| \leq 1$ that

$$
\begin{aligned}
\int_{A} \tilde{F}_{r}(\omega) d \mu(\omega) & \leq\left(\mu(A) \cdot \lambda_{i} / 2\right) \cdot 1 / 2+\mu(A)\left(1-\lambda_{i} / 2\right) \cdot 1 \\
& \leq \mu(A) \cdot\left(1-\lambda_{i} / 4\right) .
\end{aligned}
$$

This implies (1). The second part of the proposition is an immediate consequence.

In the next proposition we shall fix the program for our construction.

2.3. Proposition. There exists $F \in \sigma^{*} C\left(T, L^{\infty}\right)$ and a dense subset $D \subseteq T$ such that

(1) $\|F\|=1$ and $F_{t} \geq 0$ for $t \in T$.

(2) For $t \in T$ and $n \in \mathbf{N}$

$$
\mu\left\{F_{t} \geq 1-2^{-n}\right\} \leq 2^{-n+2} .
$$

(3) $F$ is hopeless at every $r \in D$. 
Let us admit 2.3 for the moment and show how Theorem A follows:

Proof of Theorem A. Let $F \in \sigma^{*} C\left(T, L^{\infty}\right)$ be given as in 2.3 and suppose there is some norm attaining $\bar{F} \in \sigma^{*} C\left(T, L^{\infty}\right)$ with $\|F-\bar{F}\| \leq$ 1/4. Let $\tilde{F}=\bar{F} /\|\bar{F}\| ;$ then $\|\tilde{F}\|=1$ and $\|F-\tilde{F}\| \leq 1 / 2$.

For $\alpha \in] 0,1[$ define the function

$$
\begin{aligned}
N_{\alpha}: L^{\infty} & \rightarrow \mathbf{R} \\
f & \rightarrow \sup \left\{\int_{A} f d \mu: \mu(A)=\alpha\right\},
\end{aligned}
$$

which is $\sigma^{*}$-lower semicontinuous. Applying 2.2 and assumption (2) an easy computation shows that

$$
N_{2^{-n}}\left(\tilde{F}_{r}\right) \leq 2^{-n}\left(1-2^{-n-6}\right)
$$

for $r \in D$ and so by the $\sigma^{*}$ lower semicontinuity of $N_{\alpha}$ for $r \in T$. This implies readily that, for no $r \in T, \tilde{F}_{r}$ equals 1 on a set of positive measure. As $\tilde{F}_{r} \geq-1 / 2$, for $r \in T$, we see that no $\tilde{F}_{r}$ is a norm attaining element of the unit sphere of $L^{\infty}$. In view of Proposition 1.1 this proves Theorem A.

We still have to prove 2.3 and this will involve a rather laborious construction. For preparation we need an elementary probabilistic lemma whose proof we include for the sake of completeness.

2.4. LEMMA. Let $\left(g_{n}\right)_{n=1}^{\infty}$ be an independent sequence of random variables so that $\left\|g_{n}\right\|_{\infty} \leq 1$ and $E\left(g_{n}\right)=0$. Let $\left(h_{n}\right)_{n=1}^{\infty}$ be a sequence of random variables such that $\left\|h_{n}\right\|_{\infty} \leq 1$ and each $h_{n}$ is independent of the sequence $\left(g_{k}\right)_{k=1}^{\infty}$. Then $\left(g_{n} h_{n}\right)_{n=1}^{\infty}$ tends to zero in the $\sigma^{*}$-topology of $L^{\infty}$.

Proof. Suppose not. After passing to a subsequence and changing signs, if necessary, we can find $A \in \Sigma$ and $\alpha>0$ such that, for every $n$,

$$
\int_{A} g_{n} h_{n} d \mu \geq \alpha
$$

By maybe passing to a subsequence once more we may assume (by Komlos' theorem), that the Cesàro means

$$
s_{n}=n^{-1}\left(g_{1} h_{1}+\cdots+g_{n} h_{n}\right)
$$

converge almost surely to a random variable $s$. Of course

$$
\int_{A} s d \mu \geq \alpha
$$


Hence there is $\beta>0$ such that the set $B=\{s>\beta\}$ has positive measure. $B$ is independent of every $g_{n}$ hence for every $n$

$$
\int_{B} g_{n} h_{n} d \mu=E\left(g_{n}\right) \cdot E\left(h_{n} \cdot \chi_{B}\right)=0,
$$

which implies that

$$
\int_{B} s d \mu=0
$$

the required contradiction.

Proof of Proposition 2.3. Define for $k=0,1,2, \ldots$,

$$
S_{k}=\left\{q \in \left[0,1\left[: \text { there is } l \leq k \text { s.t. } q=n_{1}^{-1}+\cdots+n_{l}^{-1}\right\} \cup\{0\},\right.\right.
$$

where $n_{i}$ are supposed to be natural numbers. It is plain to check that

$-S_{0} \subseteq S_{1} \subseteq S_{2} \subseteq \ldots$

- $S_{k}$ is compact. $S_{k}$.

$-S_{k}^{\prime}=S_{k-1}$, i.e. $S_{k-1}$ consists precisely of the accumulation points of

$-\cup_{k=0}^{\infty} S_{k}=\mathbf{Q} \cap[0,1[$.

We shall also prepare for $k \in \mathbf{N}$ partitions $Q^{k}=\left(A_{i}^{k}\right)_{i=1}^{2^{k}}$ of $\Omega$ into sets of $\mu$-measure $2^{-k}$ and for $q \in \mathbf{Q} \cap[0,1[, \lambda \in \mathbf{Q} \cap] 0,1]$ and $n \in \mathbf{N}$ a "biased Rademacher function" $B R_{n}^{q, \lambda}$ such that $B R_{n}^{q, \lambda}$ equals $-(1-\lambda)$ with probability $\lambda /(2-\lambda)$ and equals $\lambda / 2$ with probability $(2-2 \lambda) /(2-\lambda)$.

The sequence of partitions $\left(\mathbb{Q}^{k}\right)_{k=1}^{\infty}$ and the family of random variables $\left\{B_{n}^{q, \lambda}: q \in \mathbf{Q} \cap[0,1[, \lambda \in \mathbf{Q} \cap] 0,1], n \in \mathbf{N}\right\}$ are supposed to be all independent.

Now we shall construct inductively a sequence $\left(F^{k}\right)_{k=1}^{\infty}$ in $\sigma^{*} C\left(T, L^{\infty}\right)$ satisfying the following

Induction Hypotheses. $t \in T$.

(a) $\left\|F^{k}\right\|=\sup \left\{\left\|F_{s}^{k}\right\|_{L^{\infty}}: s \in S_{k} \backslash S_{k-1}\right\}=1-2^{-k-1}$ and $F_{t}^{k} \geq 0$ for

(b) For $t \in T$ and $0 \leq n \leq k-1$

$$
\mu\left\{F_{t}^{k} \geq 1-2^{-n}\right\} \leq 2^{-n+2}-2^{-k} .
$$

(c) $F^{k}$ is $S_{k}$-hopeless at every $r \in S_{k-1}$ and, for $l<k, F_{r}^{k}=F_{r}^{l}$ for all $r \in S_{l}$.

(d) For all $t \in \mathbf{Q} \cap\left[0,1\left[, F_{t}^{k}\right.\right.$ is a simple function with rational coefficients and measurable with respect to the $\sigma$-algebra generated by the partitions $\left\{\mathbb{Q}^{l}, l=1, \ldots, k\right\}$ and the random variables $\left\{B_{n}^{q, \lambda}: q \in S_{k-1}\right.$, $\lambda \in \mathbf{Q} \cap] 0,1], n \in \mathbf{N}\}$. 
(e) $F_{t}^{k}$ is locally Lipschitzian with respect to $\|\cdot\|_{\infty}$ on $T \backslash S_{k-1}$, i.e. for $t \in T \backslash S_{k-1}$ there is a constant $L^{k}(t)$ such that for $t^{\prime} \in T,\left|t-t^{\prime}\right|<$ $L^{k}(t)^{-1}$ we have

$$
\left\|F_{t}^{k}-F_{t^{\prime}}^{k}\right\|_{\infty} \leq L^{k}(t) \cdot\left|t-t^{\prime}\right| .
$$

(f) $\mu\left\{F_{t}^{k} \neq F_{t}^{k-1}\right\} \leq 2^{-k} \forall t \in T$.

To start the induction define $F^{0}$ by letting $F_{t}^{0}$ be identically the constant function $1 / 2$. For the convenience of the reader and in order to make the idea of construction clearer we carry out the first induction step, which is technically simpler, before passing to the general one.

Step 1. A: Definition of $F^{1}$ on $S_{0}$ : Let $F_{0}^{1}=F_{0}^{0}$.

B: Definition of $F^{1}$ on $S_{1} \backslash S_{0}$ : We define

$$
F_{s}^{1}=F_{s}^{0}+B R_{n}^{0,1 / 2} \cdot \chi_{A_{1}^{1}}
$$

if $s \in S_{1} \backslash S_{0}$ is of the form $s=n^{-1}$ for odd $n$ and

$$
F_{s}^{1}=F_{s}^{0}+B R_{n}^{0,1 / 2} \cdot \chi_{A_{2}^{1}}
$$

if $s \in S_{1} \backslash S_{0}$ is of the form $s=n^{-1}$ for even $n$.

C: Definition of $F^{1}$ on $T \backslash S_{1}$ : For every $s \in S_{1} \backslash S_{0}$ we define $\varepsilon(s)=$ $\operatorname{dist}\left(s, S_{1} \backslash\{s\}\right) / 2$, which is strictly positive and rational. Let $t \in T \backslash S_{1}$; if there is a (necessarily unique) $s \in S_{1} \backslash S_{0}$ such that $|t-s|<\varepsilon(s)$ then we define

$$
F_{t}^{1}=F_{t}^{0}+(1-|t-s| / \varepsilon(s))\left[F_{s}^{1}-F_{s}^{0}\right] .
$$

If there is no such $s$ we define simply

$$
F_{t}^{1}=F_{t}^{0} \text {. }
$$

It is plain to check that (a), (b), (d), (e) and (f) are satisfied for $F^{1}$. Let us show (c): $F^{1}$ is $S_{1}$-hopeless at 0 . Write $F_{0}^{1}$ as

$$
F_{0}^{1}=\sum_{i=1}^{2}(1-1 / 2) \cdot \chi_{A_{i}^{1}} .
$$

Fix $A \subseteq A_{i}^{1}$, say $A \subseteq A_{1}^{1}$. Then

$$
\begin{aligned}
\lim _{n \rightarrow \infty} \mu\left\{A \cap\left\{B R_{n}^{0,1 / 2}=-1 / 2\right\}\right\} & =\mu(A) \cdot \mu\left\{B R_{n}^{0,1 / 2}=-1 / 2\right\} \\
& =\mu(A) \cdot 1 / 3 .
\end{aligned}
$$

Hence if $s=n^{-1}$ where $n$ is large and odd

$$
\mu\left\{\omega \in A: F_{s}^{1}=0\right\}>1 / 4 \cdot \mu(A),
$$

which readily shows that $F^{1}$ is $S_{1}$-hopeless at 0 . 
Finally we have to show that $F^{1}$ is $\sigma^{*}$-continuous. In view of (e) we have to check the $\sigma^{*}$-continuity only at 0 . Let $\left(t_{j}\right)_{j=1}^{\infty} \subseteq T$ tend to 0 . By passing to a subsequence we may suppose that for $s \in S_{1} \backslash S_{0}$, there is at most one $t_{j}$ which lies in ] $-\varepsilon(s), s+\varepsilon(s)$. Then the sequence $F_{t}^{1}-F_{0}^{1}$ is of the form described in Lemma 2.4: It is the product of some $B_{n_{i}}^{0,1 / 2}$ with a scalar multiple (less or equal to one) of either $\chi_{A_{1}^{1}}$ or $\chi_{A_{2}^{1}}$; so we may conclude that $F_{t_{j}}^{1}-F_{0}^{1}$ tends $\sigma^{*}$ to zero, i.e. $F^{1}$ is $\sigma^{*}$-continuous at $t=0$.

Step k. Suppose $F^{1}, \ldots, F^{k-1}$ and $L^{k-1}(t)$ defined.

A: Definition of $F^{k}$ on $S_{k-1}$ : Let $F_{r}^{k}=F_{r}^{k-1}$ for $r \in S_{k-1}$.

B: Definition of $F^{k}$ on $S_{k} \backslash S_{k-1}$ : First fix $r \in S_{k-1} \backslash S_{k-2}$ and write

$$
F_{r}^{k-1}=\sum_{i=1}^{m(r)}\left(1-\lambda_{\imath}^{r}\right) \chi_{B_{\imath}^{r}}
$$

where $\left\{B_{i}^{r}\right\}_{l=1}^{m(r)}$ is a partition of $\Omega$, belonging to the $\sigma$-algebra generated by $\left\{Q^{l}: l<k\right\}$ and $\left\{B_{n}^{q, \lambda}: q \in S_{k-2}\right\}$ and where the $\lambda_{i}^{r}$ are rational coefficients with $2^{-k} \leq \lambda_{i}^{r} \leq 1$. Now find a strictly positive rational number $\delta(r)$, such that

$$
\delta(r) \leq \min \left\{\operatorname{dist}\left(r, S_{k-1} \backslash\{r\}\right), L^{k-1}(r)^{-1} \cdot \min \left\{\lambda_{i}^{r}, 1-\lambda_{i}^{r}: \lambda_{i}^{r}<1\right\}\right\} .
$$

Note that the intervals \{]$r, r+\delta(r)\left[: r \in S_{k-1} \backslash S_{k-2}\right\}$ are mutually disjoint.

Now fix $s \in S_{k} \backslash S_{k-1}$ : if $s$ is of the form $s=r+n^{-1}$ for some $r \in S_{k-1} \backslash S_{k-2}$ with $n^{-1}<\delta(r)$ (in which case $r$ is unique), define

$$
F_{s}^{k}=F_{s}^{k-1}+[1-|r-s| / \delta(r)] \cdot \chi_{B_{t}^{r}} \cdot \chi_{A_{j}^{k}} \cdot B R_{n}^{r, \lambda_{t}^{r}}
$$

if $n$ equals $i+(j-1) m(r)$ modulo $2^{k} m(r)$, where $1 \leq j \leq 2^{k}$ and $1 \leq i$ $\leq m(r)$.

If $s \in S_{k} \backslash S_{k-1}$ is not of the above form, define simply $F_{s}^{k}=F_{s}^{k-1}$.

C: Definition of $F^{k}$ on $T \backslash S_{k}$ : First find for each $s \in S_{k} \backslash S_{k-1}$ a strictly positive number $\varepsilon(s)$ which is smaller than $\operatorname{dist}\left(s, S_{k}\{s\}\right) / 2$ and $L^{k-1}(s)^{-1}$ multiplied by the minimum of the $\lambda$ 's and $(1-\lambda)$ 's, where $\lambda$ runs through the (finitely many) values different form zero, which $F_{s}^{k-1}$ assumes.

Note again that the intervals \{]$s-\varepsilon(s), s+\varepsilon(s)\left[: s \in S_{k} \backslash S_{k-1}\right\}$ are mutually disjoint.

Now fix $t \in T \backslash S_{k}$; if $t$ is such that there is a (necessarily unique) $s \in S_{k} \backslash S_{k-1}$ with $\left.t \in\right] s-\varepsilon(s), s+\varepsilon(s)$ [ we define

$$
F_{t}^{k}=F_{t}^{k-1}+[1-|s-t| / \varepsilon(s)] \cdot\left[F_{s}^{k}-F_{s}^{k-1}\right] .
$$

If $t$ is not of this form, define simply $F_{t}^{k}=F_{t}^{k-1}$. 
We have defined $F^{k}$ on all of $T$ and we now have to check properties (a) to (f):

(a) Let $r \in S_{k-1} \backslash S_{k-2}$; then it follows from the definition of $B^{r, \lambda r}$ and the hypothesis

$$
0 \leq F_{r}^{k-1} \leq 1-2^{-k}
$$

that for $n \in \mathbf{N}, 1 \leq i \leq m(r), 1 \leq j \leq 2^{k}$

$$
0 \leq F_{r}^{k-1}+\chi_{B_{l}^{r}} \cdot \chi_{A_{J}^{k}} \cdot B R_{n}^{r, \lambda_{l}^{r}} \leq 1-2^{-k-1} .
$$

The factor $[1-|r-s| / \delta(r)]$ is chosen small enough to get for $s \in$ $S_{k} \backslash S_{k-1}$

$$
0 \leq F_{s}^{k} \leq 1-2^{-k-1}
$$

and the factor [ $1-|s-t| / \varepsilon(s)]$ is small enough to get for $t \in T \backslash S_{k}$

$$
0 \leq F_{t}^{k} \leq 1-2^{-k-1} \text {. }
$$

Finally, in order to show that

$$
\left\|F^{k}\right\|=\sup \left\{F_{s}^{k}: s \in S_{k} \backslash S_{k-1}\right\}=1-2^{-k-1}
$$

find, for $\varepsilon>0, r \in S_{k-1} \backslash S_{k-2}$ and $\lambda_{i}^{r}$ such that $\lambda_{i}^{r}<2^{-k}+\varepsilon$. If $n$ is sufficiently large and $n$ equals $i$ modulo $2^{k}$. Then, for $s=r+n^{-1}$

$$
\left\|F_{s}^{k}\right\|_{\infty}>1-2^{-k-1}+\varepsilon .
$$

(b) and (f): Since $\mu\left(A_{j}^{k}\right)=2^{-k}$, we see that $F_{t}^{k}$ differs from $F_{t}^{k-1}$ at most on a set of measure $2^{-k}$, from which (f) and (b) follow.

(c): $F^{k}$ is $S_{k-1}$-hopeless at every $r \in S_{k-2}$ by induction hypothesis. For $r \in S_{k-1} \backslash S_{k-2}$ write

$$
F_{k}^{k-1}=F_{r}^{k}=\sum_{j=1}^{2^{k}} \sum_{i=1}^{m(r)} \lambda_{i}^{r} \chi_{\left(B_{i}^{r} \cap A_{j}^{k}\right)} .
$$

For every $A$, contained in $B_{i}^{r} \cap A_{j}^{k}$ for some $i$ and $j$, we have

$$
\lim _{n \rightarrow \infty} \mu\left\{\omega \in A: B R_{n}^{r, \lambda_{i}^{r}}=1-\lambda_{i}^{r}\right\}=\mu(A) \cdot \lambda_{i}^{r} /\left(2-\lambda_{i}^{r}\right)
$$

It follows that given $\varepsilon>0$ for $n$ large enough and equal to $i+(j-1) m(r)$ modulo $2^{k} m(r)$ we have for $s=r+n^{-1}$

$$
\mu\left\{\omega \in A:\left|F_{s}(\omega)\right| \leq \varepsilon\right\}>\mu(A) \cdot \lambda_{i}^{r} / 2 .
$$

(d) and (e): obvious.

Finally we have to show that $F^{k}$ is $\sigma^{*}$-continuous. In view of (e) we have to check the $\sigma^{*}$-continuity only at $S_{k-1}$. 
Let $\left(t_{j}\right)_{j=1}^{\infty} \in T$ tend to $s_{0} \in S_{k-1}$. By passing to a subsequence we may assume that, for each $s \in S_{k} \backslash S_{k-1}$ there is at most one $t_{j}$ which lies in $] s-\varepsilon(s), s+\varepsilon(s)[$. Hence

$$
F_{t_{j}}^{k}-F_{t_{j}}^{k-1}=c_{j} \cdot \chi_{C_{j}} \cdot B R_{k_{j}}^{s_{,}, \lambda,},
$$

where $c_{j}$ is a constant between 0 and $1, C_{j}$ is a set depending only on $\left\{B R_{k}^{q, \lambda}: q \in S_{k-2}\right\}$ and $\left\{\mathbb{Q}^{l}\right\}_{l \leq k}$ while $s_{j}$ is some element of $S_{k-1} \backslash S_{k-2}$ (and $\lambda_{j}$ and $k_{j}$ are some elements of $\left.\left.\mathbf{Q} \cap\right] 0,1\right]$ and $\mathbf{N}$ respectively). If $j \neq i$ then either $s_{j} \neq s_{i}$ or $k_{j} \neq k_{j}$; so we may apply Lemma 2.4 and conclude that $F_{t_{j}}^{k}-F_{t_{j}}^{k-1}$ tends $\sigma^{*}$ to zero. Since

$$
\sigma^{*}-\lim _{j} F_{t_{j}}^{k-1}=F_{s_{0}}^{k-1}=F_{s_{0}}^{k}
$$

we see that $F^{k}$ is indeed $\sigma^{*}$-continuous. This finishes the induction step.

It follows from (f) that the sequence $\left(F_{t}^{k}\right)_{k=1}^{\infty}$ is $\|\cdot\|_{1}$-convergent uniformly in $t \in T$, hence

$$
F_{t}=\|\cdot\|_{1}-\lim _{k \rightarrow \infty} F_{t}^{k}
$$

is a well-defined element of $\sigma^{*} C\left(T, L^{\infty}\right)$. It follows from (a), (b) and (c) of the induction hypothesis that $F$ satisfies the corresponding assumptions of 2.3 if we let $D=\mathbf{Q} \cap[0,1[$. This finishes the proof of Prop. 2.3 and therefore of Theorem A.

One may ask for which compact spaces $K$ the norm attaining operators are dense in $B\left(L^{1}, C(K)\right)$. It is easily deduced from the representation 1.1 that this is the case if $K$ has a dense set of isolated points (observed in [5]). On the other hand the above construction may be applied to some other concrete examples of compact spaces. The author has checked this, apart from $[0,1]$, for the torus, the Cantor set, a countable product of the one point compactification of $\mathbf{N}$ and for products of these compact spaces with an arbitrary compact space. This leaves of course open the question of characterising the class of compact spaces $K$ for which the norm attaining operators are dense in $B\left(L^{1}, C(K)\right)$.

3. We now pass to the proof of Theorem B. It is based on a simple lemma. In the sequel $K$ will denote a compact Hausdorff space.

3.1. LemMa. Let $W$ be a weakly compact subset of the space $\Re(K)$ of Radon measures on $K$ and let $\mu_{0} \in \mathfrak{N}(K)$.

For $\varepsilon>0$ there is an operator $S: \mathfrak{N}(K) \rightarrow \mathfrak{N}(K),\|S\|=1$ such that

(i) there is $f_{0} \in C(K),\left\|f_{0}\right\|=1$ with

$$
\left\|\mu_{0}\right\|=\left\langle f_{0}, S \mu_{0}\right\rangle \text {. }
$$

(ii) $\|S \mu-\mu\|<\varepsilon$ for all $\mu \in W$. 
Proof. Take the Hahn-decomposition $\mu_{0}=\mu_{0}^{+}-\mu_{0}^{-}$of $\mu_{0}$ into its positive and negative part and find a partition of $K$ into two Borel sets $B^{+}$ and $B^{-}$such that

$$
\mu_{0}^{+}\left(B^{+}\right)+\mu_{0}^{-}\left(B^{-}\right)=\left\|\mu_{0}\right\| .
$$

Since $V$ is weakly compact, it is uniformly inner regular ([2], Lemma VI.2.13) and we can therefore find compact subsets $K^{+}$and $K^{-}$of $B^{+}$and $B^{-}$respectively such that

$$
|\mu|\left(B^{+} \backslash K^{+}\right)+|\mu|\left(B^{-} \backslash K^{-}\right)<\varepsilon / 2,
$$

for all $\mu \in W$.

Fix points $x^{+} \in K^{+}$and $x^{-} \in K^{-}$and let $F: K \rightarrow K$ be the map

$$
F(x)= \begin{cases}x & \text { for } x \in K^{+} \cup K^{-}, \\ x^{+} & \text {for } x \in B^{+} \backslash K^{+}, \\ x^{-} & \text {for } x \in B^{-} \backslash K^{-} .\end{cases}
$$

Clearly $F$ is Borel measurable. Define $S: ~ \Re(K) \rightarrow \Re(K)$ by

$$
S(\mu)=F(\mu),
$$

$F(\mu)$ denoting the image measure of $\mu$ under $F$. Evidently $\|S\|=1$ and $\|S \mu-\mu\|<\varepsilon$, for $\mu \in W$. Find $f_{0} \in C(K),\left\|f_{0}\right\|=1$ such that $f_{0}$ equals 1 on $K^{+}$and -1 on $K^{-}$; then $\left\langle f_{0}, S \mu_{0}\right\rangle=\left\|\mu_{0}\right\|$.

Proof of Theorem B. Let $A^{*}: X^{*} \rightarrow \operatorname{\Re (}(K)$ denote the adjoint of $A$ and $W$ the image of the closed unit ball of $X^{*}$ under $A^{*}$. In view of the weak compactness of $W$ we may apply Stegall's theorem ([7],p. 176) to find an operator $T$ : $\Re(K) \rightarrow \Re(K)$ (of rank 1) such that $\|T\|<$ $\varepsilon /(2\|A\|)$ and such that $W_{1}=(\operatorname{Id}+T) W$ has an element, say $\mu_{0}$, of maximal norm, i.e.

$$
\left\|\mu_{0}\right\|=\sup \left\{\|\mu\|: \mu \in W_{1}\right\}=\left\|(\operatorname{Id}+T) A^{*}\right\| .
$$

Now we apply Lemma 3.1 to find $S: \Re(K) \rightarrow \Re(K),\|S\|=1$, such that

(i) $\sup \{\|S \mu-\mu\|: \mu \in W\} \leq \varepsilon / 2$,

(ii) there is $f_{0} \in C(K),\left\|f_{0}\right\|=1$ with

$$
\left\|\mu_{0}\right\|=\left\langle f_{0}, S \mu_{0}\right\rangle .
$$

Denote $\tilde{A}^{*}$ the operator $S(\mathrm{Id}+T) A^{*}$.

$$
\begin{aligned}
\left\|\tilde{A}^{*}-A^{*}\right\| & \leq\left\|S A^{*}-A^{*}\right\|+\left\|S T A^{*}\right\| \\
& \leq \varepsilon / 2+\|S\| \cdot\|T\| \cdot\left\|A^{*}\right\|<\varepsilon .
\end{aligned}
$$


The adjoint of $\tilde{A}^{*}$, namely $\tilde{A}^{* *}: C(K)^{* *} \rightarrow X^{* *}$ given by $\tilde{A}^{* *}=$ $A^{* *}\left(\mathrm{Id}+T^{*}\right) S^{*}$, takes $C(K)^{* *}$ into $X$, as $A^{* *}$ does so. Finally let $\tilde{A}$ : $C(K) \rightarrow X$ be the restriction of $\tilde{A}^{* *}$ to $C(K)$. It is plain to justify the above introduced notation, i.e. that $\tilde{A}^{*}$ is the adjoint of $\tilde{A}$. Also $\|\tilde{A}-A\|$ $<\varepsilon$ and

$$
\|\tilde{A}\|=\left\|\tilde{A}^{*}\right\| \leq\left\|(\mathrm{Id}+T) A^{*}\right\|
$$

On the other hand

$$
\begin{aligned}
\left\|\tilde{A}\left(f_{0}\right)\right\| & =\sup \left\{\left\langle f_{0}, S(\operatorname{Id}+T) A^{*} x^{*}\right\rangle:\left\|x^{*}\right\| \leq 1\right\} \\
& =\sup \left\{\left\langle f_{0}, S \mu\right\rangle: \mu \in W_{1}\right\} \\
& \geq\left\langle f_{0}, S \mu_{0}\right\rangle=\left\|(\operatorname{Id}+T) A^{*}\right\|
\end{aligned}
$$

which shows readily that $\left\|\tilde{A} f_{0}\right\|=\|\tilde{A}\|$.

\section{REFERENCES}

1. J. Bourgain, On dentability and the Bishop-Phelps property, Israel J. Math., 28, No. 4 (1977), 215-271.

2. J. Diestel and J. J. Uhl, Vector measures, A.M.S. Math. Surveys, vol. 15, Providence, Rhode Island, 1977.

3. A. Iwanik, Norm attaining operators on Lebesgue spaces, Pacific J. Math., 83, No. 2 (1979), 381-386.

4. J. Johnson and J. Wolfe, Norm attaining operators, Studia Math., LXV (1979), 7-19.

5. J. Lindenstrauss, On operators which attain their norm, Israel J. Math., 1 (1963), 139-148.

6. I. Namioka, Separate continuity and joint continuity, Pacific J. Math., 51, No. 2 (1974), 515-531.

7. C. Stegall, Optimization of functions on certain subsets of Banach spaces, Math. Ann., 236 (1978), 171-176.

8. J. J. Uhl, Jr., Norm attaining operators on $L^{1}[0,1]$ and the Radon-Nidodym property, Pacific J. Math., 63, No. 1 (1976), 293-300.

Received July 1, 1981.

UNIVERSITAET LINZ

ALTENBERGERSTRASSE 69

A-4045 LinZ, AUSTRIA 


\title{
PACIFIC JOURNAL OF MATHEMATICS EDITORS
}

\author{
Donald BabBitT (Managing Editor) \\ University of California \\ Los Angeles, CA 90024 \\ Hugo Rossi \\ University of Utah \\ Salt Lake City, UT 84112 \\ C. C. Moore and Arthur Ogus \\ University of California \\ Berkeley, CA 94720
}

J. DugundiI

Department of Mathematics

University of Southern California

Los Angeles, CA 90089-1113

R. FINN and H. SAMELSON

Stanford University

Stanford, CA 94305

\section{ASSOCIATE EDITORS}
R. ARENS
E. F. BECKENBACH
B. H. NeUMANN
F. WOLF
K. YosHIDA

(1906-1982)

\section{SUPPORTING INSTITUTIONS}

UNIVERSITY OF ARIZONA

UNIVERSITY OF BRITISH COLUMBIA

CALIFORNIA INSTITUTE OF TECHNOLOGY

UNIVERSITY OF CALIFORNIA

MONTANA STATE UNIVERSITY

UNIVERSITY OF NEVADA, RENO

NEW MEXICO STATE UNIVERSITY

OREGON STATE UNIVERSITY

\author{
UNIVERSITY OF OREGON \\ UNIVERSITY OF SOUTHERN CALIFORNIA \\ STANFORD UNIVERSITY \\ UNIVERSITY OF HAWAII \\ UNIVERSITY OF TOKYO \\ UNIVERSITY OF UTAH \\ WASHINGTON STATE UNIVERSITY \\ UNIVERSITY OF WASHINGTON
}

The Supporting Institutions listed above contribute to the cost of publication of this Journal, but they are not owners or publishers and have no responsibility for its content or policies.

Mathematical papers intended for publication in the Pacific Journal of Mathematics should be in typed form or offset-reproduced (not dittoed), double spaced with large margins. Please do not use built up fractions in the text of the manuscript. However, you may use them in the displayed equations. Underline Greek letters in red, German in green, and script in blue. The first paragraph must be capable of being used separately as a synopsis of the entire paper. In particular it should contain no bibliographic references. Please propose a heading for the odd numbered pages of less than 35 characters. Manuscripts, in triplicate, may be sent to any one of the editors. Please classify according to the scheme of Math. Reviews, Index to Vol. 39. Supply name and address of author to whom proofs should be sent. All other communications should be addressed to the managing editor, or Elaine Barth, University of California, Los Angeles, California 90024.

There are page-charges associated with articles appearing in the Pacific Journal of Mathematics. These charges are expected to be paid by the author's University, Government Agency or Company. If the author or authors do not have access to such Institutional support these charges are waived. Single authors will receive 50 free reprints; joint authors will receive a total of 100 free reprints. Additional copies may be obtained at cost in multiples of 50 .

The Pacific Journal of Mathematıcs is issued monthly as of January 1966. Regular subscription rate: $\$ 132.00$ a year (6 Vol., 12 issues). Special rate: $\$ 66.00$ a year to individual members of supporting institutions.

Subscriptions, orders for numbers issued in the last three calendar years, and changes of address should be sent to Pacific Journal of Mathematics, P.O. Box 969, Carmel Valley, CA 93924, U.S.A. Old back numbers obtainable from Kraus Periodicals Co., Route 100, Millwood, NY 10546.

The Pacific Journal of Mathematics ISSN 0030-8730 is published monthly by the Pacific Journal of Mathematics at P.O. Box 969, Carmel Valley, CA 93924. Application to mail at Second-class postage rates is pend ing at Carmel Valley, California, and additional mailing offices. Postmaster: Send address changes to Pacific Journal of Mathematics, P. O. Box 969, Carmel Valley, CA 93924.

PUBLISHED BY PACIFIC JOURNAL OF MATHEMATICS, A NON-PROFIT CORPORATION

Copyright $(1) 1983$ by Pacific Journal of Mathematics 


\section{Pacific Journal of Mathematics}

\section{Vol. 105, No. $2 \quad$ October, 1983}

Spiros Argyros, On compact spaces without strictly positive measure . . . . . 257

Steven Robert Bell, Regularity of the Bergman projection in certain nonpseudoconvex domains ............................273

Carlos R. Borges and Gary Fred Gruenhage, Sup-characterization of

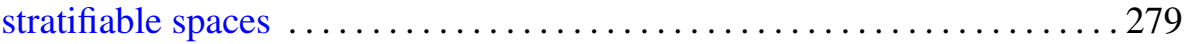

Giuseppe Ceresa and Alberto Collino, Some remarks on algebraic

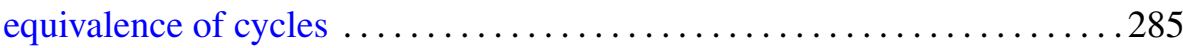

Charles Kam-Tai Chui and Maurice Hasson, Degree of uniform approximation on disjoint intervals ....................... 291

Gary Gundersen, Meromorphic functions that share two finite values with

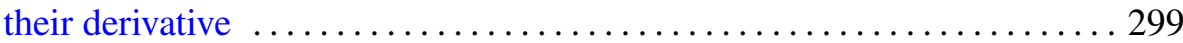

Richard I. Hartley, Lifting group homomorphisms ............... 311

Gerald William Johnson and David Lee Skoug, Notes on the Feynman integral. III. The Schroedinger equation ..................... 321

John Cronan Kieffer, Some topologies on the set of discrete stationary

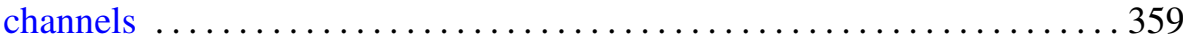

Harald Luschgy and Wolfgang Thomsen, Extreme points in the Hahn-Banach-Kantorovič setting . ............................... 387

Zbigniew Piotrowski, A. Rosłanowski and Brian M. Scott, The pinched-cube topology ................................ 399

Elias Saab and Paulette Saab, A dual geometric characterization of Banach

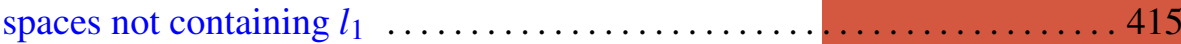

Walter Schachermayer, Norm attaining operators on some classical Banach

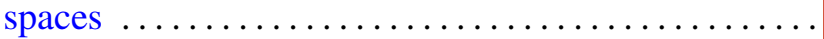

Martin Scharlemann, Essential tori in 4-manifold boundaries

Jacques C. H. Simon, Nonlinear representations of Poincaré group and global solutions of relativistic wave equations ..........

Adrian R. Wadsworth, $p$-Henselian field: $K$-theory, Galois cohomology, and graded Witt rings $\ldots \ldots \ldots \ldots \ldots \ldots \ldots \ldots \ldots$ 